\title{
HIGHER-ORDER THEORIES FOR DOUBLY CURVED LAMINATED LATTICE AND HONEYCOMB STRUCTURES
}

\author{
TORNABENE F. ${ }^{1}$, VISCOTI M. ${ }^{2}$, AND DIMITRI R. ${ }^{3}$ \\ University of Salento, Department of Innovation Engineering, Via per Monteroni, 73100, Lecce, Italy \\ a) Corresponding author: francesco.tornabene@unisalento.it \\ b) matteo.viscoti@unisalento.it \\ c) rossana.dimitri@unisalento.it
}

Key words: Doubly-Curved Shells, GDQ Method, Honeycomb, Lattice Structures.

\begin{abstract}
A collocation model based on a Generalized Differential Quadrature Method (GDQM) is proposed for the dynamic analysis of anisotropic curved laminated structures with a central lattice core and different external constraints. The theory is based on the Equivalent Single Layer (ESL) approach, together with higher-order kinematic assumptions. The reliability of the proposed method is checked with respect to classical 3D FEM-based solutions, for different shell geometries, lamination schemes and unit cell configurations. Based on the numerical investigation, the proposed formulation reveals to be computationally performing even for complicated shapes and structural members, compared to more expensive commercial finite-element-based packages.
\end{abstract}

\section{INTRODUCTION}

Optimization tasks are becoming even more important in a design process of complex structures. Doubly-curved structural members play a key role, since their shape can provide a meaningful increase in stiffness with a reduced weight. In addition, lattice multi-layered shells are becoming very popular in many engineering fields [1], such that they require advanced modelling strategies to check for the best cell layup depending on the design demand [2-3]. In a context where common optimization algorithms are based on an iterative process, simple but performing models are preferred. In order to obtain a simple, but accurate simulation, the periodicity of the structure must be exploited: the so-called Representative Volume Element (RVE) is determined first, to check for the pertaining equivalent elastic properties under some fixed load cases [4]. A large variety of homogenization procedures can be found in literature for honeycomb cells: the macromechanical stiffness matrix is analytically obtained employing various different hypotheses, like thin-walled assumptions [5], the influence of shear and axial effects [6-10], the node shape [11], as well as its rigidity [12]. According to come theories, however, elastic constants do not converge to a unique solution [13], such that an interpolation procedure must be applied between some extreme values, as suggested by Grediac [14] and Scarpa and Tolmin [15]. In any case, an interpolation method can not be generalized, since the essential constants are calibrated on a series of analyses of cells with some fixed characteristics a priori, employing the least square method. As far as grid structures is concerned, a periodic pattern can be found starting from the number of rib 
families occurring in the shell, characterized by a particular angle distribution [16-17]. The computation of the equivalent elastic properties account for the contribution of each rib family to the whole unit cell without considering coupling effects.

For the analysis of doubly-curved structures, the Equivalent Single Layer (ESL) approach is one of the best strategies, whose properties are referred to the reference mid-surface [18]. The key aspect relies on the correct choice of the through-the-thickness shape of the unknowns, in a unified setting. The geometry description of a shell structure is dictated by its curvature properties, such that any kind of structure can be defined as particular case of a doubly-curved member. Tornabene et al. [18] applied this method to anisotropic laminated shells by employing higher order theories.

For a discrete definition of the governing equations of the problem, here we apply the so called Generalized Differential Quadrature (GDQ) method, due to its capability to discretize directly the derivatives of the unknown functions, and to solve the equations in a strong form [18-19]. In the present work a bi-dimensional structural model with 3D capabilities is proposed for the dynamic study of doubly-curved structures with a lattice or honeycomb core. The procedure follows the ESL method combined with higher-order theories. The fundamental equations of the problem together with the related boundary conditions are derived from the Hamiltonian principle, whose solutions are determined computationally via the GDQ method. A modal analysis is performed systematically in the DiQuMASPAB code [20] for doubly-curved lattice and honeycomb structures, evaluating the influence of the kinematic assumptions throughout the thickness on the overall response.

\section{SHELL THEORITI \\ In this section we shells: the attention}
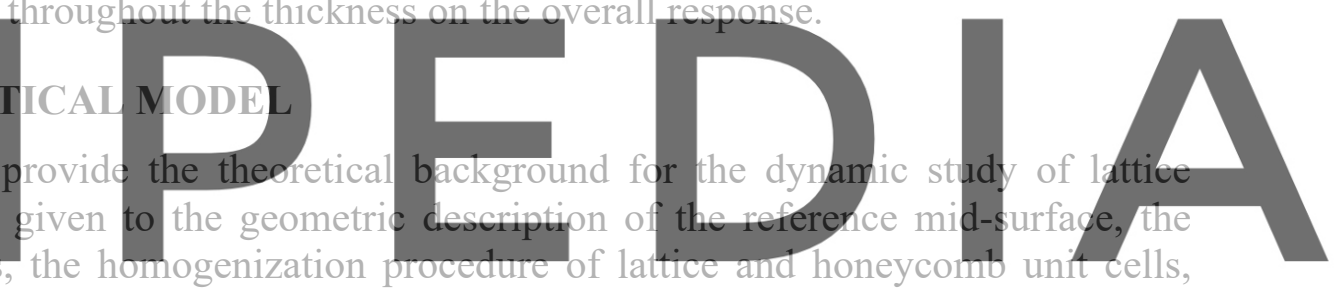

and the fundamental equations of the problem. A brief overview of the GDQ numerical

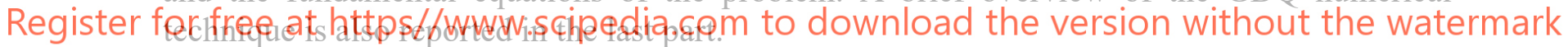

\subsection{Geometry description and unified formulation}

Shell structures can be described accurately with 3D formulations [1], especially for cases involving deep shells. However, accounting for warping phenomena occurring within laminae of a stacking sequence, it is possible to define an equivalent bi-dimensional model in which all the geometric, kinematic and mechanic quantities are referred to the mid-surface of the shell. According to the ESL method, an arbitrary position vector $\mathbf{R}\left(\alpha_{1}, \alpha_{2}, \zeta\right)$ can be referred to its projection $\mathbf{r}\left(\alpha_{1}, \alpha_{2}\right)$ on the mid-surface [18] as

$$
\mathbf{R}\left(\alpha_{1}, \alpha_{2}, \zeta\right)=\mathbf{r}\left(\alpha_{1}, \alpha_{2}\right)+\frac{h\left(\alpha_{1}, \alpha_{2}\right)}{2} z \mathbf{n}\left(\alpha_{1}, \alpha_{2}\right)
$$

where $z=2 \zeta / h\left(\alpha_{1}, \alpha_{2}\right) \in[-1,1]$ is a dimensionless variable defining an arbitrary point in the thickness direction $\alpha_{3}=\zeta$. In Eq. (1) the normal unit vector can be defined by means of the partial derivatives $\mathbf{r}_{, 1}$ and $\mathbf{r}_{, 2}$ of the reference surface position vector $\mathbf{r}\left(\alpha_{1}, \alpha_{2}\right)$ with respect to in-plane spatial coordinates $\alpha_{1}$ and $\alpha_{2}$, together with the Lamè parameters $A_{1}$ and $A_{2}$. 
The displacement field can be expressed in terms of generalized displacement components [18], following the ESL approach

$$
\left[\begin{array}{l}
U_{1} \\
U_{2} \\
U_{3}
\end{array}\right]=\sum_{\tau=0}^{N+1}\left[\begin{array}{ccc}
F_{\tau}^{\alpha_{1}} & 0 & 0 \\
0 & F_{\tau}^{\alpha_{2}} & 0 \\
0 & 0 & F_{\tau}^{\alpha_{3}}
\end{array}\right]\left[\begin{array}{l}
u_{1}^{(\tau)} \\
u_{2}^{(\tau)} \\
u_{3}^{(\tau)}
\end{array}\right] \Leftrightarrow \quad \mathbf{U}=\sum_{\tau=0}^{N+1} \mathbf{F}_{\tau} \mathbf{u}^{(\tau)}
$$

The accuracy of the model depends on the choice of a proper set of functions $F_{\tau}^{\alpha_{i}}, i=1,2,3$. If $l$ is the total number of laminae within a laminate, the overall thickness of the shell is defined as

$$
h\left(\alpha_{1}, \alpha_{2}\right)=\sum_{k=1}^{l} h_{k}\left(\alpha_{1}, \alpha_{2}\right)
$$

The three principal curvilinear coordinates $\alpha_{i}, i=1,2,3$ must be constrained to predefined intervals, i.e. $\alpha_{i} \in\left[\alpha_{i}^{0}, \alpha_{i}^{1}\right]$, for $i=1,2$; whereas $\zeta=\alpha_{3}\left(\alpha_{1}, \alpha_{2}\right) \in\left[-h\left(\alpha_{1}, \alpha_{2}\right) / 2, h\left(\alpha_{1}, \alpha_{2}\right) / 2\right]$. Thus, the thickness of an arbitrary $k$-th lamina is defined as $h_{k}=\zeta_{k+1}-\zeta_{k}$, where $\zeta_{k}$ and $\zeta_{k+1}$ stand for its extreme $\alpha_{3}$ coordinate values at the bottom and top sides.

Even though the axiomatic assumption (2) is capable to account for several warping effects, when the shell is obtained from the superimposition of different laminae, a complex

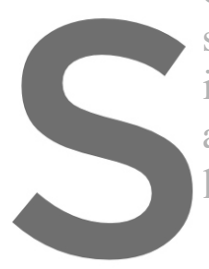
set of internal actions improper definition of the assumption, at the $N$ leading to
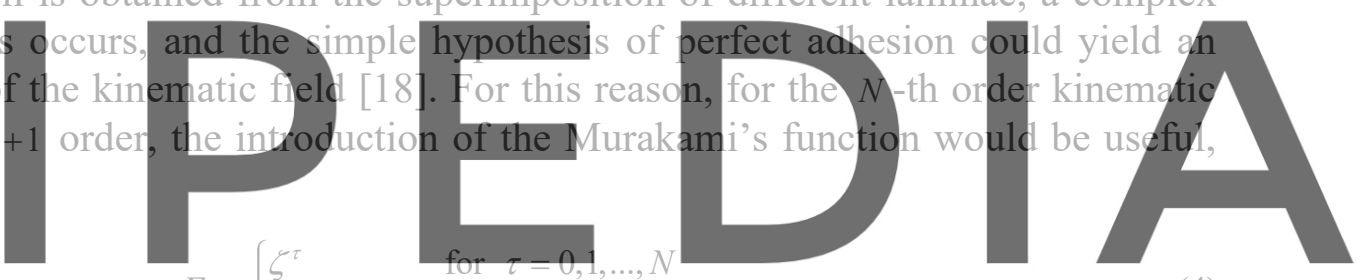

Register for free at httos//www scipedia $(-1)^{k} z_{k}$ for $\tau=N+1$

(4)

where the dimensionless parameter $z_{k}=z_{k}(\zeta) \in[-1,1]$ is defined as

$$
z_{k}=\frac{2}{\zeta_{k+1}-\zeta_{k}} \zeta-\frac{\zeta_{k+1}+\zeta_{k}}{\zeta_{k+1}-\zeta_{k}}
$$

The strain field is then derived from the partial derivatives with respect to the reference coordinates $\alpha_{1}, \alpha_{2}, \alpha_{3}$ of the position vector (1). In a compact matrix notation, we get

$$
\varepsilon=\mathbf{D U}
$$

It is useful to split the differential operator $\mathbf{D}$ of Eq. (6) in different parts, such that $\mathbf{D}_{\zeta}$ is the through-the-thickness geometric matrix, whereas $\mathbf{D}_{\Omega}^{\alpha_{i}}$ is related to the $i$-th displacement field component vector

$$
\boldsymbol{\varepsilon}=\mathbf{D} \mathbf{U}=\mathbf{D}_{\zeta}\left(\sum_{i=1}^{3} \mathbf{D}_{\Omega}^{\alpha_{i}}\right) \mathbf{U}
$$

By combination of Eq. (2) with Eq. (7), we get the following definition for the generalized 
strain field $\varepsilon^{(\tau)}\left(\alpha_{1}, \alpha_{2}, t\right)=\left[\begin{array}{lllllllll}\varepsilon_{1}^{(\tau)} & \varepsilon_{1}^{(\tau)} & \gamma_{1}^{(\tau)} & \gamma_{2}^{(\tau)} & \gamma_{13}^{(\tau)} & \gamma_{23}^{(\tau)} & \omega_{13}^{(\tau)} & \omega_{23}^{(\tau)} & \varepsilon_{3}^{(\tau)}\end{array}\right]^{T}[18]$

$$
\boldsymbol{\varepsilon}=\mathbf{D} \mathbf{U}=\sum_{\tau=0}^{N+1} \mathbf{D}_{\zeta}\left(\sum_{i=1}^{3} \mathbf{D}_{\Omega}^{\alpha_{i}}\right) \mathbf{F}_{\tau} \mathbf{u}^{(\tau)}=\sum_{\tau=0}^{N+1} \mathbf{D}_{\zeta}\left(\sum_{i=1}^{3} F_{\tau}^{\alpha_{i}} \mathbf{D}_{\Omega}^{\alpha_{i}}\right) \mathbf{u}^{(\tau)}=\sum_{\tau=0}^{N+1} \sum_{i=1}^{2} \mathbf{Z}^{(\tau) \alpha_{i}} \mathbf{D}_{\Omega}^{\alpha_{i}} \mathbf{u}^{(\tau)}=\sum_{\tau=0}^{N+1} \mathbf{Z}^{(\tau)} \boldsymbol{\varepsilon}^{(\tau)}
$$

For each $\tau$-th expansion order, the strain vector is defined as $\boldsymbol{\varepsilon}^{(\tau) \alpha_{i}}=\mathbf{D}_{\Omega}^{\alpha_{i}} \mathbf{u}^{(\tau)}$ for $\tau=0,1,2, \ldots, N, N+1, \alpha_{i}, i=1,2,3$. For detailed treatment, we refer to the work by Tornabene et al. [18].

\subsection{Constitutive behavior}

In the present paper, the constituent materials of the shell are assumed to be linear elastic. Since the geometric configuration of the lattice core unit cell comes into the definition of various material symmetries, the model is developed for the case of completely anisotropic medium for each lamina. For each $k$-th layer it is possible to define the anisotropic

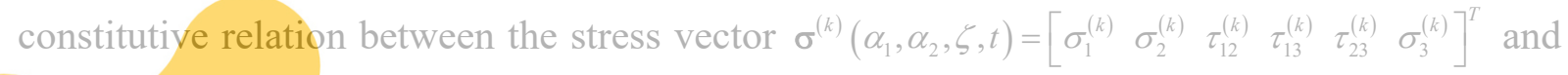
the strain one $\varepsilon^{(k)}\left(\alpha_{1}, \alpha_{2}, \zeta, t\right)=\left[\begin{array}{lllllll}\varepsilon_{1}^{(k)} & \varepsilon_{2}^{(k)} & \gamma_{12}^{(k)} & \gamma_{13}^{(k)} & \gamma_{23}^{(k)} & \varepsilon_{3}^{(k)}\end{array}\right]^{T}$, by means of the equivalent stiffness matrix $\overline{\mathbf{C}}^{(k)}$, i.e.

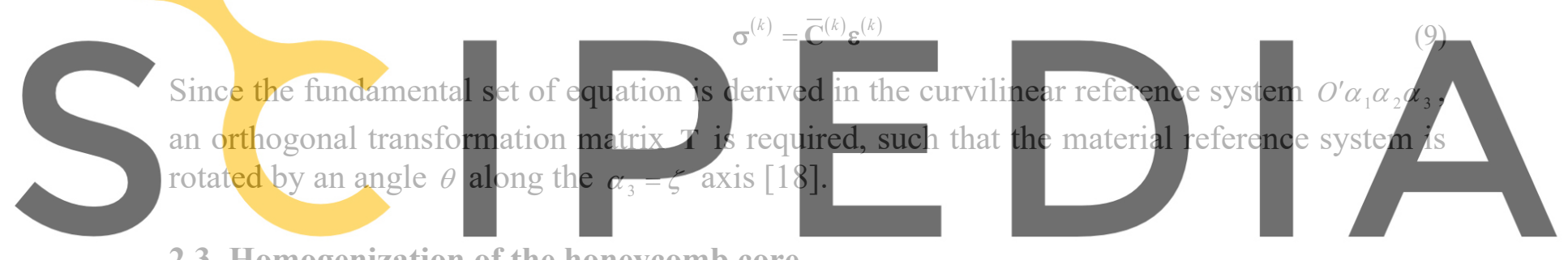

2.3 Homogenization of the honeycomb core

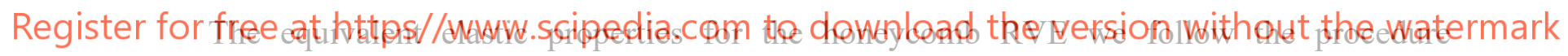

suggested by Sorohan et al. [6-7], which account for the influence of shear stress

distributions. The equivalent elastic properties depend strongly on the geometrical cell layup rather than constituent material. For this reason, it is common to refer to the cell slenderness instead of the actual geometric parameter. With reference to Figure 1a, three dimensionless cell quantities are introduced, namely $\alpha=l_{2} / l, \beta=s / l$ and $\gamma=b / l$. Considering some elementary independent load cases (LCs), the stiffness of a general honeycomb can be expressed in terms of the engineering constants [7]. If we denote with the superscript $s$ all the mechanical characteristics of the raw material, one gets:

$$
\rho=\rho_{s} \frac{\beta(\alpha+2)}{2 \cos \vartheta(\alpha+\sin \vartheta)}
$$




$$
\begin{gathered}
E_{1}=k_{1} E_{s} \beta^{3} \frac{\cos \vartheta}{(\alpha+\sin \vartheta) \sin ^{2} \vartheta}, \quad E_{2}=k_{2} E_{s} \beta^{3} \frac{\alpha+\sin \vartheta}{\cos ^{3} \vartheta}, \quad E_{3}=E_{s} \frac{\rho}{\rho_{s}} \\
G_{12}=k_{12} E_{s} \beta^{3} \frac{\alpha+\sin \vartheta}{\alpha^{2}(1+2 \alpha) \cos \vartheta}, \quad G_{13}=G_{s} \beta \frac{\cos \vartheta}{\alpha+\sin \vartheta}, \quad v_{12}=c_{12} \frac{\cos ^{2} \vartheta}{(\alpha+\sin \vartheta) \sin \vartheta} \\
G_{23}^{L}=G_{s} \beta \frac{\alpha+\sin \vartheta}{(1+2 \alpha) \cos \vartheta}, \quad G_{23}^{U}=G_{s} \beta \frac{\alpha+2 \sin ^{2} \vartheta}{2(\alpha+\sin \vartheta) \cos \vartheta}, \quad v_{31}=v_{32}=v_{s} \\
c_{12}=k_{1}\left(1+\beta^{2}\left(1.4+1.5 v_{s}\right)\right), \quad k_{1}=1 /\left(1+\beta^{2}\left(2.4+1.5 v_{s}+\cot ^{2} \vartheta\right)\right) \\
k_{2}=1 /\left(1+\beta^{2}\left(2.4+1.5 v_{s}+\tan ^{2} \vartheta+2 \alpha / \cos ^{2} \vartheta\right)\right) \\
k_{12}=1 /\left(1+2 \alpha_{b}+\beta_{b}^{2}\left(\frac{2.4+1.5 v_{s}}{\alpha_{b}}(4+\alpha+\sin \vartheta)+\frac{\alpha+\sin \vartheta}{\alpha_{b}^{2}}\left((\alpha+\sin \vartheta) \tan ^{2} \vartheta+\sin \vartheta\right)\right)\right)
\end{gathered}
$$

As far as $G_{23}$ elastic modulus is concerned, LCs provide only an interval of variation $\left[G_{23}^{L}, G_{23}^{U}\right]$ for this constant, starting from a static and kinematic admissible solution of the problem. Grediac [14] proposed a linear interpolation between the two extreme values $G_{23}^{L}$ and $G_{23}^{U}$. Nevertheless, in the present article a non-linear interpolation based on the cell slenderness is suggested, to ensure a great accuracy for a wider range of cases

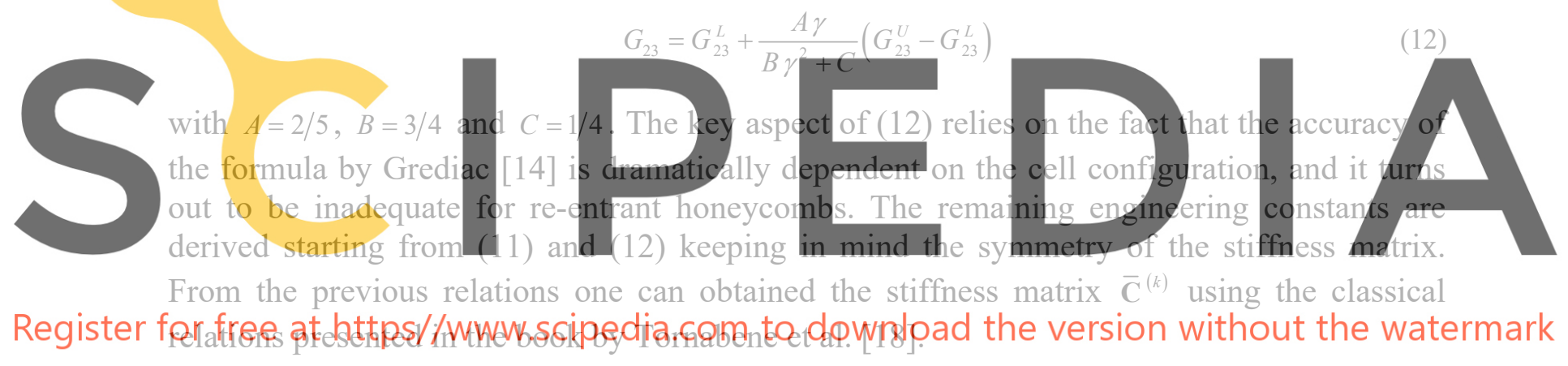

\subsection{Homogenization of the Lattice Layer}

Since the material properties are defined in the material reference system of the single rib, each frame is oriented with an angle $\phi_{i}$ with respect to the geometric reference system. If we consider the $i$-th rib of the RVE, the stress and strain vectors are defined as

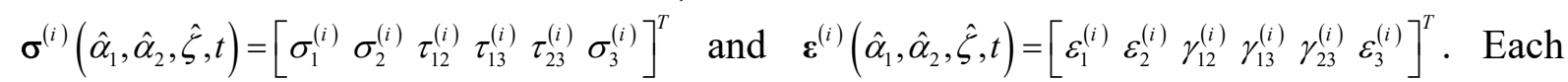
elastic constant $C_{i j}^{(i)}$ of the ribs does not consider possible coupling effects due to the presence of other frames [16-17]. The single contribution of the $i$-th rib is defined assuming an isotropic behavior of the single rib. The material elastic modulus for the $i$-th frame is labeled with $E_{i}$, whereas the Poisson'r ratio is denoted as $v_{i}$. According to Figure 1b, the relative density of the unit cell can be computed starting from the interspace $a_{i}$ between two adjacent equally-oriented beams with $\delta_{i}$ width; $\rho_{i}$, instead, denotes the density of the $i$-th frame in the unit cell 


$$
\rho=\sum_{i=1}^{n} \rho_{i} \frac{\delta_{i}}{a_{i}}
$$

If the repetitive pattern of the grid is obtained from a number of $n$ frame families, the homogenized material properties can be derived by rotating each rib of an angle equal to its inclination $\phi_{i}[16-17]$

$$
\begin{aligned}
& C_{11}^{(k)}=\sum_{i=1}^{n} E_{i} \frac{\delta_{i}}{a_{i}} \cos ^{4} \phi_{i}, C_{22}^{(k)}=\sum_{i=1}^{n} E_{i} \frac{\delta_{i}}{a_{i}} \sin ^{4} \phi_{i}, C_{33}^{(k)}=\sum_{i=1}^{n} E_{i} \frac{\delta_{i}}{a_{i}}, C_{13}^{(k)}=C_{23}^{(k)}=C_{36}^{(k)}=0 \\
& C_{12}^{(k)}=C_{66}^{(k)}=\sum_{i=1}^{n} E_{i} \frac{\delta_{i}}{a_{i}} \sin ^{2} \phi_{i} \cos ^{2} \phi_{i}, C_{16}^{(k)}=\sum_{i=1}^{n} E_{i} \frac{\delta_{i}}{a_{i}} \cos ^{3} \phi_{i} \sin \phi_{i}, C_{26}^{(k)}=\sum_{i=1}^{n} E_{i} \frac{\delta_{i}}{a_{i}} \cos \phi_{i} \sin ^{3} \phi_{i} \\
& C_{44}^{(k)}=\sum_{i=1}^{n} G_{i} \frac{\delta_{i}}{a_{i}} \cos ^{2} \phi_{i}, C_{55}^{(k)}=\sum_{i=1}^{n} G_{i} \frac{\delta_{i}}{a_{i}} \sin ^{2} \phi_{i}, C_{45}^{(k)}=\sum_{i=1}^{n} G_{i} \frac{\delta_{i}}{a_{i}} \cos \phi_{i} \sin \phi_{i}
\end{aligned}
$$

When the grid unit cells are taken in account, it is useful to introduce a compact nomenclature to identify the geometric characteristics of the RVE layup. In the present work, a single cell is denoted with the array S/F $\left[\left(\phi_{i}\right)_{a}^{\delta}\right] i=1, \ldots, n$. The symbol S/F denotes Star (S) or Flake (F) cells,

depending on the layout of the actual rib family with respect to the center of the RVE.
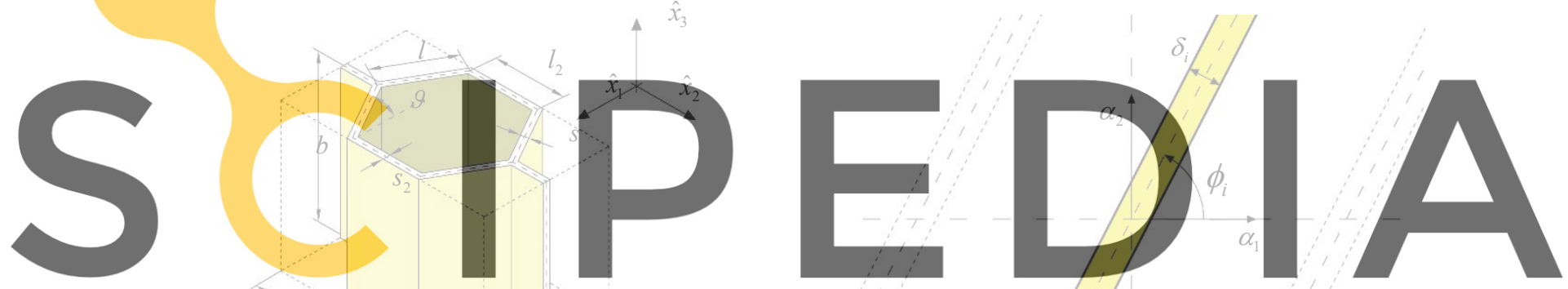

Register for free at https//www.scipedia.com to download the/version without the watermark

(a)

(b)

Figure 1: Unit cell geometric configuration: honeycomb cell (a) and grid pattern (b).

\subsection{Fundamental equations}

Once the homogenized elastic stiffness matrix of each cell unit has been written in the geometric reference system by means of the transformation matrix $\mathbf{T}$, it is possible to write the equilibrium equations by employing the Hamiltonian principle. The proposed approach is oriented to a modal analysis of shell structures with innovative-materials. Therefore, the elastic strain energy will be computed, together with the kinetic energy, involving the inertial effects.

It is useful to define generalized stress resultants from the integration along the $\alpha_{3}$-direction of the shell. The target is to rearrange the constitutive law (9) in terms of the generalized strains $\varepsilon^{(\tau) \alpha_{i}}\left(\alpha_{1}, \alpha_{2}, t\right)$. From the equivalence of the elastic strain energy with the actual stresses 
and the generalized ones, keeping in mind the elastic behavior (9) rotated in the geometrical reference system, we get [18]

$$
\mathbf{S}^{(\tau) \alpha_{i}}=\sum_{\eta=0}^{N+1} \sum_{j=1}^{3} \mathbf{A}^{(\tau \eta) \alpha_{i} \alpha_{j}} \boldsymbol{\varepsilon}^{(\eta) \alpha_{j}} \text { for } \tau=0,1,2, \ldots, N, N+1, \quad \alpha_{i}=\alpha_{1}, \alpha_{2}, \alpha_{3}
$$

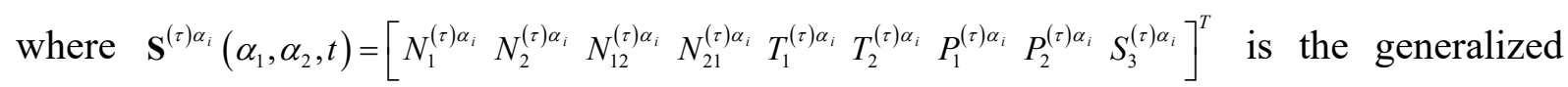
stress component vector, and $\mathbf{A}^{(\tau \eta) \alpha_{i} \alpha_{j}}$ the generalized stiffness matrix component. Based on an ESL approach (2), Eq. (15) is expressed in terms of the degrees of freedom $\mathbf{u}^{(\tau)}$ of the problem. The equivalent stiffness constants $\mathbf{A}^{(\tau \eta) \alpha_{i} \alpha_{j}}$ are computed as follows, being $\tau, \eta=0,1,2, \ldots, N, N+1, n, m=1,2,3,4,5,6, p, q=0,1,2, \alpha_{i}, \alpha_{j}=\alpha_{1}, \alpha_{2}, \alpha_{3}[18]$

$$
\begin{aligned}
& A_{n m(p q)}^{(\tau \eta) \alpha_{i} \alpha_{j}}=\sum_{k=1}^{l} \int_{\zeta}^{\zeta_{k+1}} \bar{B}_{n m}^{(k)} F_{\eta}^{\alpha_{j}} F_{\tau}^{\alpha_{i}} \frac{H_{1} H_{2}}{H_{1}^{p} H_{2}^{q}} d \zeta \quad A_{n m(p q)}^{(\tilde{z} \eta) \alpha_{\alpha} \alpha_{j}}=\sum_{k=1}^{l} \int_{\zeta}^{\zeta_{k+1}} \bar{B}_{n m}^{(k)} F_{\eta}^{\alpha_{j}} \frac{\partial F_{\tau}^{\alpha_{i}}}{\partial \zeta} \frac{H_{1} H_{2}}{H_{1}^{p} H_{2}^{q}} d \zeta \\
& \sum_{k=1}^{l} \int_{\zeta_{k}}^{\zeta_{k+1}} \bar{B}_{n m}^{(k)} \frac{\partial F_{\eta}^{\alpha_{j}}}{\partial \zeta} F_{\tau}^{\alpha_{j}} \frac{H_{1} H_{2}}{H_{1}^{p} H_{2}^{q}} d \zeta \quad A_{n m(p q)}^{(i \bar{\eta}) \alpha_{i} \alpha_{j}}=\sum_{k=1}^{l} \int_{\zeta_{k}}^{\zeta_{k+1}} \bar{B}_{n m}^{(k)} \frac{\partial F_{\eta}^{\alpha_{j}}}{\partial \zeta} \frac{\partial F_{\tau}^{\alpha_{i}}}{\partial \zeta} \frac{H_{1} H_{2}}{H_{1}^{p} H_{2}^{q}} d \zeta
\end{aligned}
$$

Coefficients $\bar{B}_{n m}^{(k)}$ can be intended as reduced elastic constants $\bar{C}_{n m}^{(k)}$ of the material, as well as their correspondent reduced ones $\bar{Q}_{n m}^{(k)}$ for the employment of the classical first order theories. In the latter case, the introduction of the shear correction factor $\kappa=5 / 6$ is compulsory.

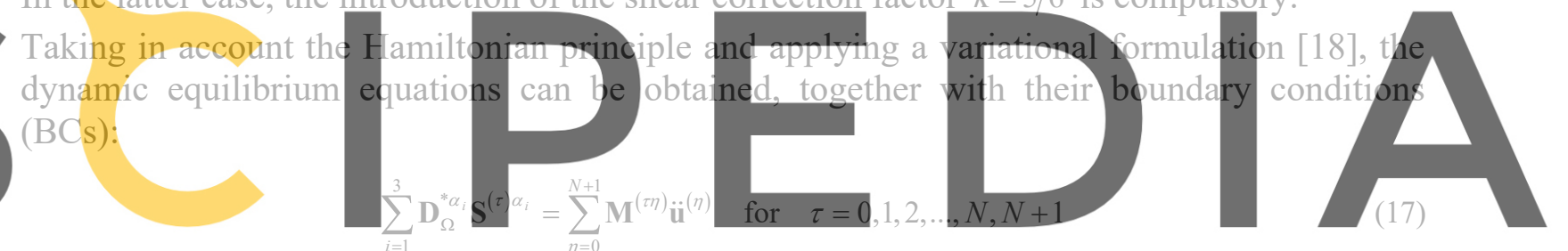

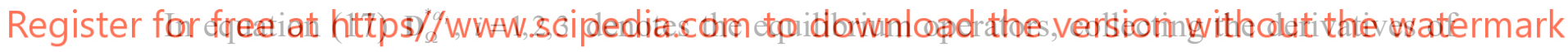

the geometric parameters in each principal direction of the shell. In addition, $\mathbf{M}^{(m)}$ stands for the generalized mass matrix [18], with $\tau, \eta=0,1,2, \ldots, N, N+1$. If $\rho^{(k)} k=1, \ldots, l$ is the density for the $k$-th lamina, the general term $I^{(\tau \eta) \alpha_{i} \alpha_{j}}$ inside the mass matrix $\mathbf{M}^{(\tau \eta)}$ is defined as

$$
I^{(\tau \eta) \alpha_{i} \alpha_{j}}=\sum_{k=1}^{l} \int_{\zeta_{k}}^{\zeta_{k+1}} \rho^{(k)} F_{\tau}^{\alpha_{i}} F_{\eta}^{\alpha_{j}} H_{1} H_{2} d \zeta \quad \text { for } \quad \alpha_{i}, \alpha_{j}=\alpha_{1}, \alpha_{2}, \alpha_{3}
$$

Based on the ESL definition of $\mathbf{S}^{(\tau) \alpha_{i}}, i=1,2,3$ for each $\tau$-th order, from the dynamic equilibrium equation (17) we can easily derive the fundamental relations for an anisotropic generally-shaped shell structure, $\mathbf{L}^{(\tau \eta)}$ being the fundamental matrix

$$
\sum_{\eta=0}^{N+1} \mathbf{L}^{(\tau \eta)} \mathbf{u}^{(\eta)}=\sum_{\eta=0}^{N+1} \mathbf{M}^{(\tau)} \ddot{\mathbf{u}}^{(\eta)} \quad \text { for } \tau=0,1,2, \ldots, N, N+1
$$

The complete expressions of constants $L_{i j}^{(\tau) \alpha_{i} \alpha_{j}}$ with $i, j=1,2,3$ and $\tau, \eta=0, \ldots, N, N+1$ are reported in the book by Tornabene et al. [18]. In order to determine the natural frequencies and mode shape of the structure, the harmonic solutions of (19) should be obtained. For this 
reason, the unknown field $\mathbf{u}^{(\tau)}$ with $\tau=0,1,2, \ldots, N, N+1$ is set in a trigonometric form, so that $\mathbf{u}^{(\tau)}\left(\alpha_{1}, \alpha_{2}, t\right)=\overline{\mathbf{U}}^{(\tau)}\left(\alpha_{1}, \alpha_{2}\right) e^{i \omega t}$. The fundamental equations (19) become

$$
\sum_{\eta=0}^{N+1} \mathbf{L}^{(\tau \eta)} \overline{\mathbf{U}}^{(\eta)}+\omega^{2} \sum_{\eta=0}^{N+1} \mathbf{M}^{(\tau \eta)} \overline{\mathbf{U}}^{(\eta)}=\mathbf{0} \quad \text { for } \tau=0,1,2, \ldots, N, N+1
$$

Based on the procedure proposed by Tornabene et al. [18] for the definition of BCs, the following nomenclature is applied to identify the shell edges: West (W), South (S), East (E) and North (N) parts are such that $\alpha_{1}^{0} \leq \alpha_{1} \leq \alpha_{1}^{1}, \quad \alpha_{2}=\alpha_{2}^{0}$ (W), $\quad \alpha_{1}=\alpha_{1}^{1}, \quad \alpha_{2}^{0} \leq \alpha_{2} \leq \alpha_{2}^{1}$ (S), $\alpha_{1}^{0} \leq \alpha_{1} \leq \alpha_{1}^{1}, \quad \alpha_{2}=\alpha_{2}^{1}(\mathrm{E}), \alpha_{1}=\alpha_{1}^{0}, \quad \alpha_{2}^{0} \leq \alpha_{2} \leq \alpha_{2}^{1}(\mathrm{~N})$.

\subsection{Numerical Implementation}

The governing equations (20) are here discretized according to the GDQ method [18]. If $f(x)$ is an arbitrary smooth function in a closed interval, its $n$-th order derivative is defined as

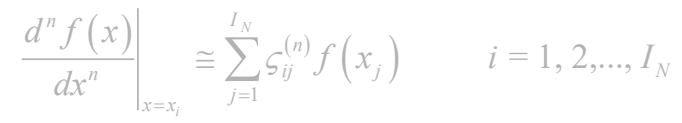

where $I_{N}$ is the total number of grid points, and the coefficients $\varsigma_{i j}^{(n)}$ are computed following a recursive formulation. The grid has been built following the Chebyshev-Gatiss-Lobatto
distribution [18]. Based on Eq. (21) we can discretize Eq. (20), thus obtaining the following
compact relation
\[ K \delta=\omega^{2} \mathbf{M} \delta \]

static condensation of (22) is applied, so that only inner points DOFs are explicated [18]

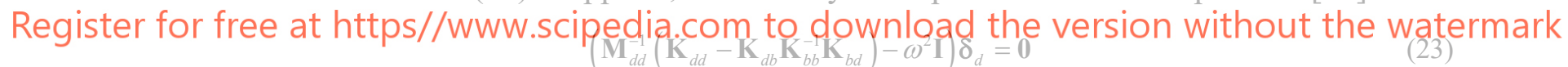

\section{APPLICATIONS AND RESULTS}

The above-mentioned theoretical framework is here validated for some case studies by comparing the first five natural frequencies of different shell structures as provided by the 2D GDQ model with predictions from refined 3D FEM simulations, here assumed as reference solutions. Then, some completely-curved structures are investigated, and a parametric analysis is performed for grid and honeycomb layers. The first set of analyses consists of a comparative evaluation between the frequencies provided by the GDQ theory and 3D FEM model (Figure 2). All the proposed examples start assuming an isotropic raw material ( $\left.E_{s}=70 \mathrm{GPa}, v_{s}=0.33, \rho_{s}=2700 \mathrm{~kg} / \mathrm{m}^{3}\right)$. The homogenization (10)-(11) of the honeycomb layer is then validated by performing a modal analysis for a rectangular plate with the proposed approach and 3D FEM-based simulations. The results are provided in Table 1. It can be seen that for all investigated examples a great agreement between FEM and GDQ occurs, even though in the latter case a reduced number of DOFs is strictly required to get accurate 
solutions. In addition, the accuracy of the honeycomb homogenized model is not affected by the geometric parameter issues, primarily by the internal angle $\vartheta$ and the layer height.

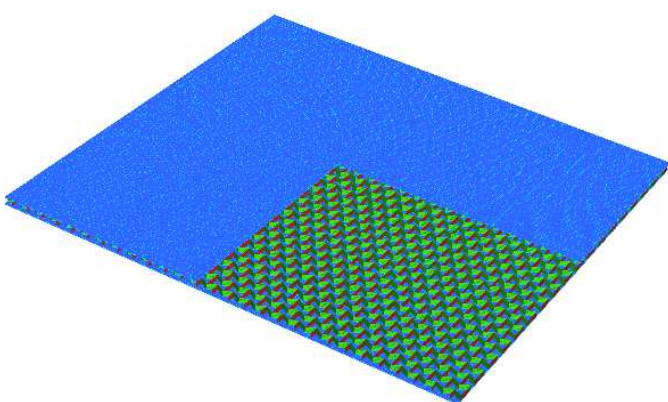

(a)

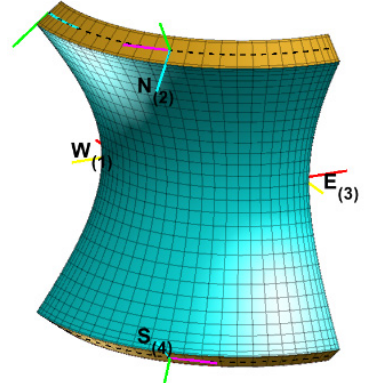

(b)

Figure 2: Refined 3D FEM model of a rectangular plate with a honeycomb infill (a) and homogenized revolution hyperbolic hyperboloid model reinforced with a grid layer (b).

TABLE 1: Modal analysis of a rectangular laminated plate with a honeycomb layer.

CCCC Rectangular Plate
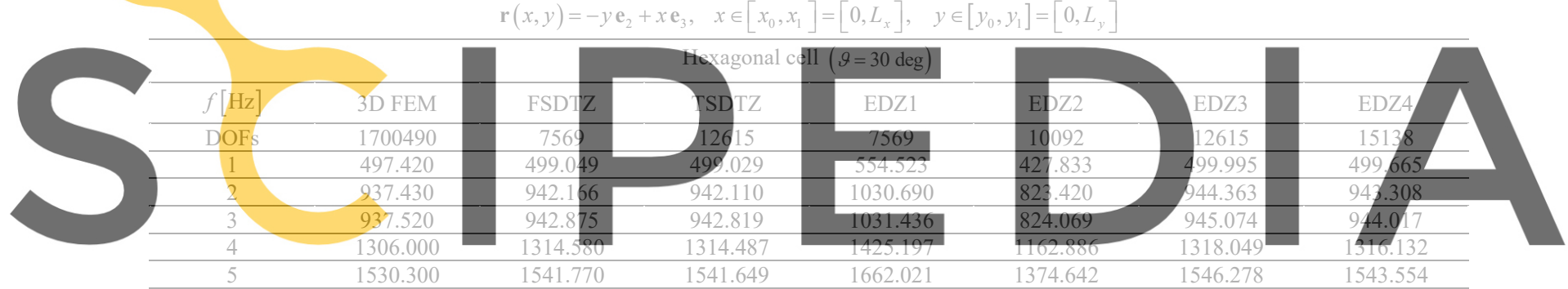

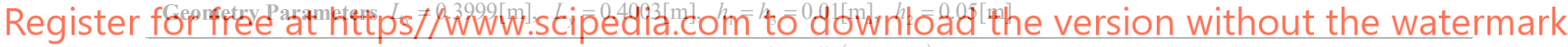
Rectangular cell $(\vartheta=0 \mathrm{deg}$

\begin{tabular}{|c|c|c|c|c|c|c|c|}
\hline$f[\mathrm{~Hz}]$ & 3D FEM & FSDTZ & TSDTZ & EDZ1 & EDZ2 & EDZ3 & EDZ4 \\
\hline DOFs & 1938258 & 7569 & 12615 & 7569 & 10092 & 12615 & 15138 \\
\hline 1 & 489.070 & 490.427 & 490.408 & 542.534 & 422.777 & 491.460 & 491.072 \\
\hline 2 & 907.740 & 910.100 & 910.051 & 989.235 & 802.364 & 912.576 & 911.288 \\
\hline 3 & 917.610 & 923.906 & 923.853 & 1006.364 & 812.050 & 926.137 & 924.976 \\
\hline 4 & 1264.300 & 1271.842 & 1271.760 & 1371.416 & 1133.618 & 1275.503 & 1273.322 \\
\hline 5 & 1468.000 & 1472.951 & 1472.848 & 1577.546 & 1325.986 & 1477.901 & 1474.691 \\
\hline \multicolumn{8}{|c|}{ Geometry Parameters $L_{x}=0.4[\mathrm{~m}], L_{y}=0.4[\mathrm{~m}], \quad h_{1}=h_{3}=0.01[\mathrm{~m}], \quad h_{2}=0.05[\mathrm{~m}]$} \\
\hline \multicolumn{8}{|c|}{ Re-entrant cell $(\vartheta=-30 \mathrm{deg})$} \\
\hline$f[\mathrm{~Hz}]$ & 3D FEM & FSDTZ & TSDTZ & EDZ1 & EDZ2 & EDZ3 & EDZ4 \\
\hline DOFs & 1224690 & 7569 & 12615 & 7569 & 10092 & 12615 & 15138 \\
\hline 1 & 490.960 & 490.972 & 490.954 & 540.360 & 426.029 & 492.155 & 491.673 \\
\hline 2 & 901.600 & 906.894 & 906.847 & 980.996 & 804.838 & 909.522 & 908.038 \\
\hline 3 & 911.940 & 907.855 & 907.808 & 981.960 & 805.786 & 910.503 & 909.009 \\
\hline 4 & 1249.600 & 1250.808 & 1250.733 & 1340.796 & 1124.447 & 1254.864 & 1252.265 \\
\hline 5 & 1444.900 & 1454.799 & 1454.703 & 1550.972 & 1318.759 & 1460.028 & 1456.391 \\
\hline \multicolumn{8}{|c|}{ Geometry Parameters $L_{x}=0.3999[\mathrm{~m}], L_{y}=0.4003[\mathrm{~m}], h_{1}=h_{3}=0.01[\mathrm{~m}], h_{2}=0.05[\mathrm{~m}]$} \\
\hline \multicolumn{8}{|c|}{ Cell Layup $l=8.1407[\mathrm{~mm}], l_{2}=15.0218[\mathrm{~mm}], s=s_{2}=0.1[\mathrm{~mm}]$} \\
\hline
\end{tabular}


In Tables 2-3 we summarize the first five natural frequencies here computed for fully clamped shells with different geometries and curvatures considering a central core made of honeycomb patterns and grid frames, respectively. Different cell layups are investigated, accounting for a possible variation of the layer height. The analysis is tackled with different HSDTs, embedding the Murakami's function (4).

TABLE 2: Modal analysis of doubly curved structures with different honeycomb cell configurations and external constraints. Each model is based on a grid made of $I_{N} \times I_{M}=31 \times 31$ points, requiring 15138 DOFs.

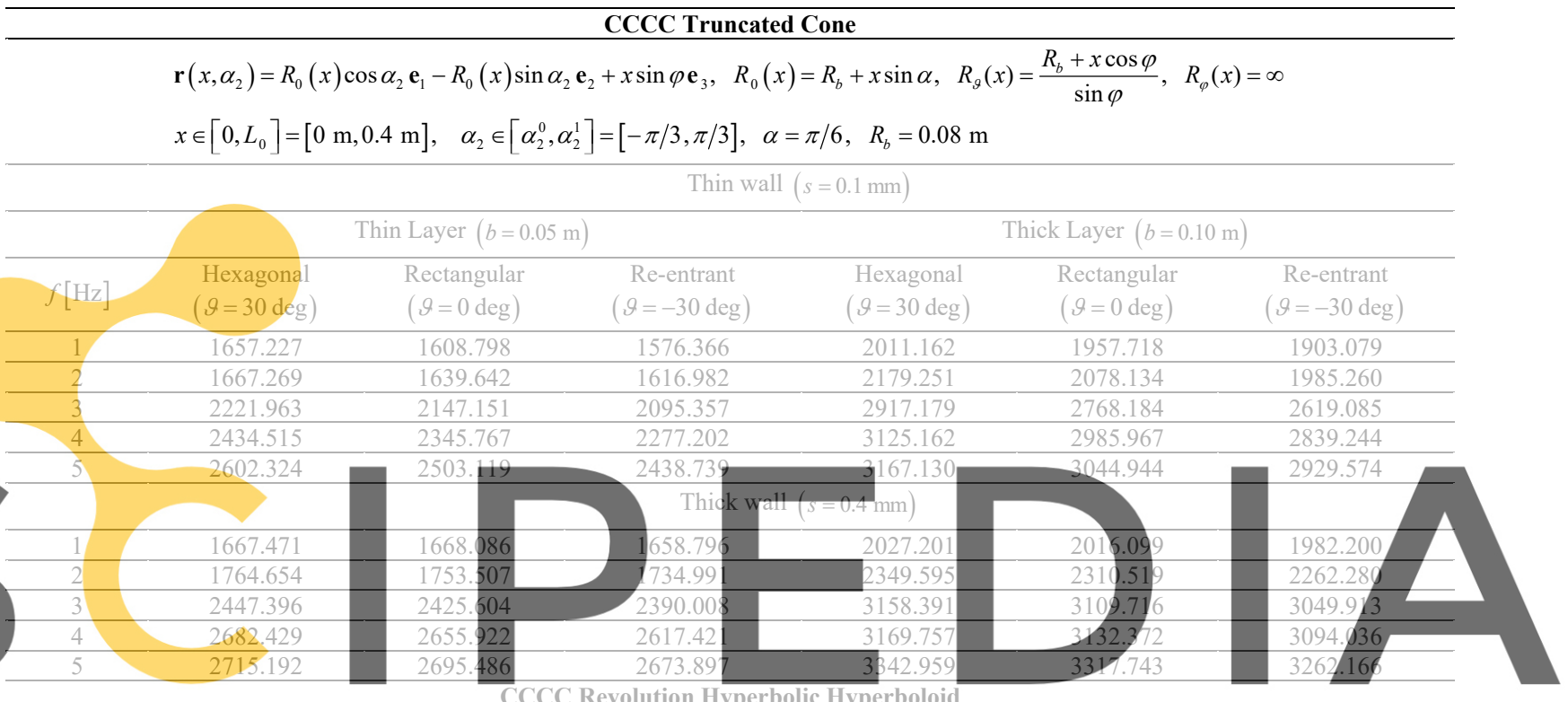

$\mathrm{r}\left(\alpha_{1}, \alpha_{2}\right)=a \cosh \alpha_{1} \cos \alpha_{2} \mathrm{e}_{1}-a \cosh \alpha_{1} \sin \alpha_{2} \mathrm{e}_{2}+c \sinh \alpha_{1} \mathrm{e}$

Register for free at https//WWW.scipedia.com to download the version without the watermark

\begin{tabular}{|c|c|c|c|c|c|c|}
\hline 1 & 4131.112 & 4014.426 & 3906.901 & 4844.136 & 4681.732 & 4463.809 \\
\hline 2 & 4198.343 & 4112.187 & 4020.120 & 4869.505 & 4689.504 & 4.503 .004 \\
\hline 3 & 4646.210 & 4494.957 & 4324.393 & 5316.217 & 5234.266 & 5028.628 \\
\hline 4 & 4682.749 & 4554.176 & 4389.052 & 5537.462 & 5350.350 & 5059.643 \\
\hline \multirow[t]{3}{*}{5} & 5354.179 & 5240.959 & 5111.220 & 6054.736 & 5850.609 & 5588.587 \\
\hline & \multicolumn{6}{|c|}{ Thick wall $(s=0.4 \mathrm{~mm})$} \\
\hline & \multicolumn{3}{|c|}{ Thin Layer $(b=0.05 \mathrm{~m})$} & \multicolumn{3}{|c|}{ Thick Layer $(b=0.10 \mathrm{~m})$} \\
\hline 1 & 4500.867 & 4487.545 & 4420.049 & 5302.710 & 5228.929 & 5119.306 \\
\hline 2 & 4608.481 & 4560.647 & 4483.242 & 5477.890 & 5394.412 & 5197.384 \\
\hline 3 & 5022.580 & 5107.134 & 4999.194 & 5526.572 & 5649.525 & 5412.585 \\
\hline 4 & 5340.633 & 5344.049 & 5215.148 & 5864.442 & 6043.142 & 5897.546 \\
\hline 5 & 5847.358 & 5810.670 & 5667.187 & 7069.135 & 7000.055 & 6717.297 \\
\hline
\end{tabular}

Geometric Layup $l=8.1407[\mathrm{~mm}], l_{2}=15.0218[\mathrm{~mm}]$ 
TABLE 3: Modal analysis of a revolution hyperbolic hyperboloid with a central grid core employing different HSDT theories.

\begin{tabular}{|c|c|c|c|c|c|c|}
\hline \multicolumn{7}{|c|}{ CCCC Revolution Hyperbolic Hyperboloid } \\
\hline \multicolumn{4}{|c|}{$\mathbf{r}\left(\alpha_{1}, \alpha_{2}\right)=a \cosh \alpha_{1} \cos \alpha_{2} \mathbf{e}_{1}-a \cosh \alpha_{1} \sin \alpha_{2} \mathbf{e}_{2}+c \sinh \alpha_{1} \mathbf{e}_{3}$} & $\left.x_{1}^{1}\right]=[-1,1]$, & $\left.\alpha_{2}^{0}, \alpha_{2}^{1}\right]=[0, \pi / 2]$, & $a=c=0.5 \mathrm{~m}$ \\
\hline \multicolumn{7}{|c|}{ Thin Layer $(b=0.05 \mathrm{~m})$} \\
\hline$f[\mathrm{~Hz}]$ & FSDTZ & TSDTZ & EDZ1 & EDZ2 & EDZ3 & EDZ4 \\
\hline DOFs & 7569 & 12615 & 7569 & 10092 & 12615 & 15138 \\
\hline 1 & 1171.606 & 1186.549 & 1208.034 & 1179.045 & 1194.845 & 1190.332 \\
\hline 2 & 1289.555 & 1301.213 & 1316.268 & 1295.383 & 1307.409 & 1304.120 \\
\hline 3 & 1420.729 & 1464.369 & 1484.147 & 1435.767 & 1481.836 & 1471.330 \\
\hline 4 & 1434.474 & 1471.590 & 1485.532 & 1447.074 & 1486.304 & 1477.559 \\
\hline 5 & 1548.062 & 1576.195 & 1598.883 & 1558.876 & 1588.857 & 1581.717 \\
\hline \multicolumn{7}{|c|}{ Thick Layer $(b=0.10 \mathrm{~m})$} \\
\hline 1 & 1323.700 & 1357.564 & 1359.981 & 1337.761 & 1374.209 & 1362.464 \\
\hline 2 & 1358.916 & 1378.572 & 1383.111 & 1368.241 & 1389.483 & 1381.879 \\
\hline 3 & 1663.839 & 1729.824 & 1702.673 & 1680.953 & 1751.206 & 1734.890 \\
\hline 4 & 1715.224 & 1792.931 & 1766.397 & 1737.477 & 1820.117 & 1799.678 \\
\hline 5 & 1789.544 & 1854.351 & 1839.681 & 1809.927 & 1879.373 & 1861.378 \\
\hline Cell Layu & ${ }_{0.141}^{0.010} / 0_{0.100}^{0.010} /$ & $\left.0_{0.100}^{0.010}\right]$ & & & & \\
\hline
\end{tabular}

\section{CONCLUSIONS}

A theoretical model is here proposed for the dynamic analysis of curved surfaces reinforced with lattice layers and honeycomb cores. The geometrical issues rely on the differential geometry, and the kinematic field is described within a unified formulation. The equations of motion are derived from the Hamiltonian principle, accounting for the possibility of treating a completely anisotropic honeycomb cell and lattice structure. The stiffness matrix is computed by means of a RVE homogenization technique, whereby the fundamental set of equations is discretized with the GDQ method. Some validation case studies are presented, in which natural frequencies from the homogenized model are compared to refined FEM-based predictions from commercial codes. After that, some completely doubly-curved structures are analyzed by employing different HSDTs, as well as geometries and cell layups, with very accurate results even with a reduced computational cost.

\section{REFERENCES}

[1] Reddy J.N., Mechanics of laminated composite plates and shells, CRC Press, Boca Raton, 2003.

[2] Vasiliev V.V., Razin A.F., Anisogrid composite lattice structures for spacecraft and aircraft applications, Composite Structures, 76 (2006), 182-189.

[3] Vasiliev V.V., Evgeny V.M., Mechanics and analysis of composite materials, Elsevier, Oxford, 2001.

[4] Kalamkarov A.L., Andrianov I.V., Danishevsâ V.V., Asymptotic homogenization of composite materials and structures, Applied Mechanics Reviews 62 (2009), 030802.

[5] Gibson L.J., Ashby M.F., Cellular solids: structure and properties, Cambridge University Press, Cambridge, 1999. 
[6] Sorohan S., Sandu M., Constantinescu D.M., Sandu A. G., On the evaluation of mechanical properties of honeycombs by using finite element analyses, INCAS Bulletin, 7 (2015), 135-150.

[7] Sorohan S., Sandu M., Constantinescu D.M., Sandu A.G., Estimation of out of plane Shear Moduli for honeycomb cores with Modal Finite Element Analyses, The Romanian Journal of Technical Sciences, Applied Mechanics, 61 (2016), 71-88.

[8] Sorohan S., Constantinescu D.M. Sandu M., Sandu A.G., On the homogenization of hexagonal honeycombs under axial and shear loading. Part I: analytical formulation for free skin effect, Mechanics of Materials, 119 (2018), 74-91.

[9] $\mathrm{Fu}$ M.-H., Yin J.R., Equivalent elastic parameters of the honeycomb core, Acta Mechanica Sinica, 15 (1999), 113-118.

[10] Torabi K., Afshari H., Aboutalebi F.H., Vibration and flutter analyses of cantilever trapezoidal honeycomb sandwich plates, Journal of Sandwich Structures \& Materials, 21 (2019), 2887-2920.

[11] Malek S., Gibson, L., Effective elastic properties of periodic hexagonal honeycombs, Mechanics of Materials, 91 (2015), 226-240.

[12] Sorohan S., Constantinescu D.M., Sandu M., Sandu A.G., On the homogenization of hexagonal honeycombs under axial and shear loading. Part II: comparison of free skin and rigid skin effects on effective core properties, Mechanics of Materials, 119 (2018), 92-108.

[13] Kelsey S., Gellatly R., Clark B., The shear modulus of foil honeycomb cores, Aircraft Engineering and Aerospace Technology, 30 (1958), 294-302.

[14] Grediac M., A finite element study of the transverse shear in honeycomb cores, International Journal of Solids and Structures, 30 (1993), 1777-1788.

[15] Scarpa F., Tomlin P., On the transverse shear modulus of negative Poisson ratio honeycomb structures, Fatigue \& Fracture of Engineering Materials \& Structures, 23 (2000), 717-720.

[16] G.I. Pshenichnov, A Theory of Latticed Plates and Shells, World Scientific Publishing, London, 1993.

[17] Tornabene F., Dimitri R., Higher-Order Modelling of Anisogrid Lattice Shell Structures with Complex Geometries, 18th International Conference of Numerical Analysis and Applied Mathematics (ICNAAM2020) Proceedings, 17-23 September 2020, Rhodes, Greece.

[18] Tornabene F., Bacciocchi M., Anisotropic Doubly-Curved Shells. Higher-Order Strong and Weak Formulations for Arbitrarily Shaped Shell Structures, Esculapio, Bologna, 2018.

[19] Tornabene F., Fantuzzi N., Ubertini F., Viola E., Strong formulation finite element method based on differential quadrature: a survey, Applied Mechanics Reviews, 67 (2015), 020801.

[20] F. Tornabene, N. Fantuzzi, M. Bacciocchi, DiQuMASPAB: Differential Quadrature for Mechanics of Anisotropic Shells, Plates, Arches and Beams, Società Editrice Esculapio, Bologna, 2018. 